\title{
Global Cancer Education
}

\author{
Amr S. Soliman ${ }^{1} \cdot$ Robert M. Chamberlain ${ }^{1,2}$
}

Accepted: 28 May 2021 / Published online: 12 June 2021

(c) American Association for Cancer Education 2021

Cancer is becoming a global health problem that attracts major resources from high-income countries in innovations for early detection, tailored and effective therapy, disease control, and improving quality of quality of life of the increasing number of cancer survivors. Cancer will continue to be an increasingly challenging health problem in low- and middle-income countries (LMICs) because of the increasing cancer incidence and mortality rates due to increased longevity of populations, changing lifestyles and epidemiologic and nutritional transitions, and improving diagnostic facilities for identifying cancer, late-stage presentations, and needs for palliative care for cancer survivors.

Because of the importance of cancer as a global health problem, this supplement of the Journal of Cancer Education is focused on global cancer education from different angles related to professional, patient, and public education. The interest in international cancer education in the USA started when the American Association for Cancer Education (AACE), as the main professional association for cancer education in the USA, partnered with the European Association for Cancer Education (EACE) more than four decades ago. You'll find in this supplement an editorial that addresses this partnership as the origin of the AACE global program. Over the past four decades, the two associations (AACE and EACE) have hosted the joint annual conferences that addressed different aspects related to global cancer education. The two associations also publish the Journal of Cancer Education (JCE), with

Amr S. Soliman

asoliman@med.cuny.edu

1 City University of New York School of Medicine, 160 Convent Avenue- Harris Hall 313, New York, NY 10031, USA

2 The University of Texas M.D. Anderson Cancer Center, Houston, TX, USA members from the two associations as the editorial board of the journal. The EACE started the interest in cancer education in some LMICs because of cancer education research in Africa by some of its members and the University of Groningen and University of Vienna summer courses that hosted students from different LMICs. The AACE expanded its global expansion beyond Europe when the two authors of this editorial introduced their M.D. Anderson Cancer Center's collaboration in North Africa through professional education for the epidemiologic study of inflammatory breast cancer in North Africa with the Division of Cancer Epidemiology and Genetics of the National Cancer Institute in 1999. Additional global collaboration and connections were added to the AACE portfolio after the initiation of the Cancer Epidemiology Education in Special Populations (CEESP), the Program that comprises a major component in this supplement. This supplement includes manuscripts on opportunities and future directions for capacity building for global cancer research. The authors of the manuscript from the National Cancer Institute discuss the importance of sustaining a trained workforce skilled to pursue global cancer research. Efforts to overcome gaps in this field by the US National Institutes of Health are also addressed in this manuscript. The supplement also includes a manuscript on the ECHO Project that highlights the importance of capacity building through sharing of information for professional education through telemedicine. The importance of online distance education is illustrated in this manuscript that addresses an important and timely topic for distance learning in professional cancer education.

The supplement also includes manuscripts on the development, current status, and outcomes of the CEESP Program. Because of the importance of disseminating knowledge and experiences in cancer education, this supplement includes a manuscript on the development, adjustments, and maintenance of the CEESP infrastructure and its availability for utilization by other groups 
and institutions for cancer research training and education. The supplement also includes manuscripts on the value of mentoring in cancer education and the CEESP Program. Finally, the supplement includes examples illustrating different established and growing professional, patient, and public education programs in Egypt, Oman, Morocco, and Tanzania. Lessons learned from the different experiences presented in this supplement are summarized and suggested future directions are outlined.

Publisher's Note Springer Nature remains neutral with regard to jurisdictional claims in published maps and institutional affiliations. 